\title{
La Fiscalite Congolaise Face Aux Enjeux De La Decentralisation: Cas De La Province De L'ituri De 2016 A 2018
}

\author{
Menga Yaosuwa*, Imani Gaya \\ The University of BUNIA in Acronym UNIBU
}

DOI: $10.36348 /$ sb.2019.v05i11.004 $\quad$ | Received: 21.10 .2019 | Accepted: 28.10 .2019 | Published: 13.11 .2019

*Corresponding author: Menga Yaosuwa

\section{Abstract}

Our study focuses on Congolese taxation in the face of the challenges of decentralization: the case of the province of Ituri from 2016 to 2018. We analyze the feasibility of the law regarding withholding tax by decentralized territorial entities. Thus, this study focuses on the following questions: is the withholding of revenue by the decentralized territorial entities carried out according to the spirit of the law in the matter for their development? If not, what are the consequences and possible solutions for the province of Ituri? The constant is such that withholding suffers from a systematic violation in the province of Ituri. The consequences are enormous and, moreover, too visible. This province is in an economic dependence on the central power, which consequently hinders its development. The solution to consider is compliance with article 175 of the constitution of the Democratic Republic of Congo which states that: "The share of national revenue allocated to the provinces is established at $40 \%$. It is withheld at source. To verify, apprehend and guide this study, the documentary technique is probably for us more practical, explicit and appropriate in the realization of our modest work.

Keywords: Fiction, Issues, Decentralization, Province, Rertocession, Law

Copyright @ 2019: This is an open-access article distributed under the terms of the Creative Commons Attribution license which permits unrestricted use, distribution, and reproduction in any medium for non-commercial use (NonCommercial, or CC-BY-NC) provided the original author and source are credited.

\section{SYNTHESE}

Depuis son instauration, la loi sur la retenue à la source de $40 \%$ des recettes à caractère national par les provinces souffre d'une violation systématique, surtout sur le point abordant la retenue à la source par les ETD de $40 \%$. Cette retenue ne s'effectue pas, partout dans le pays. Une situation qui s'explique par une absence brillante de volonté politique du pouvoir central.

Les conséquences sont énormes et d'ailleurs, trop visibles. Les ETD se trouvent dans une dépendance économique au pouvoir central, chose qui, conséquemment, freine leur développement. Pour y remédier, le pouvoir central devrait veiller de bonne foi à la transparence dans la rétrocession des recettes dues aux ETD. Par ailleurs, tout en soutenant l'autonomie budgétaire de chaque province, nous estimons que, l'article 175 de la constitution est loin d'être mise en application. Pour rappel, il précise que: «La part des recettes à caractère national allouées aux provinces est établie à $40 \%$. Elle est retenue à la source. »Ce qui pour nous a comme conséquence, le freinage de relance économique des nouvelles Provinces de la RDC dont l'Ituri, qui ne reçoivent pas suffisamment des moyens du Pouvoir Central pour entamer le fameux concept de «écollage ».

\section{ETAT DE LA QUESTION}

L'état de la question n'est rien d'autre que le fait pour un chercheur de consulter les travaux de ses prédécesseurs qui ont abordé quasiment le même thème que lui. Ainsi, les questions liées à la fiscalité congolaise face aux enjeux de la décentralisation, la retenue à la source de $40 \%$, la rétrocession et ou encore l'impact de la décentralisation en RDC ont intéressé une multitude des cherches ces derniers années; chacun abordant dans un angle spécifique. Pour le présent travail, nous relevons entre autres les travaux de:

Lubunga Mwindulwa $\mathrm{H}$ [1], ce chercheur se pose la question de savoir, dans son mémoire intitulé «l'impact de la décentralisation territoriale sur le développement en $R D C »$ à quel point la 
décentralisation de la RDC telle que prévue par la Constitution de la R.D.C. peut vraiment contribuer au développement de ce pays. Il pense que, avec des finances fébriles, des pratiques de mauvaise gouvernance accentuée, la République Démocratique du Congo n'est pas en mesure de développer ses entités territoriales décentralisées. C'est ainsi qu'il conclut en disant que les entités territoriales décentralisées peuvent toutefois y arriver sans bonne gouvernance.

Niyibizi M [2], celui-ci, dans son mémoire parlant de: «entraves à la mise en auvre de la décentralisation financière en République Démocratique du Congo ». Il a épinglé, comme des obstacles majeurs au développement, les faits suivants: l'égoïsme, la corruption, le détournement du denier public et l'impunité dans le chef des autorités tant au niveau national que local. Il préconise que les autorités qui animent les E.T.D soient élues par la population afin qu'elles se sentent redevables, au lieu d'être nommées car, selon lui, la nomination les pousse à dilapider les ressources financières de leurs entités dans la corruption des autorités qui les nomment à leurs fonctions respectives afin de se maintenir au pouvoir.

Nkongolo Musungula [3], dans son étude, il pense qu'il y a lieu d'actualiser la législation financière, d'appliquer de manière correcte la rétrocession en faveur des provinces et de rendre opérationnelle la péréquation pour réduire les déséquilibres insoutenables entre provinces.

Parlant des provinces les moins nanties, la retenue à la source de leurs maigres recettes à caractère national équivaudrait à une formule de financement, à titre principal, de leur développement par leur propre misère et se traduit, in fine, en une limitation insoutenable des capacités et perspectives d'autonomie locale, conclut-il.

Dans notre étude, nous aurons cette plénitude d'analyser l'application de la loi quant à la retenue à la source de $40 \%$, chose qui démarquera notre travail à ceux des autres. Une étude qui a un penchant peu spécifique, à savoir « la fiscalité congolaise face aux enjeux de la décentralisation: cas de la province de l'Ituri de 2016 à 2018 ».

\section{PROBLÉMATIQUE}

De façon générale, la problématique est définie comme une structure d'informations dont la mise en relation engendre chez le chercheur un constat d'écart et se traduisant par un effet de surprise ou de questionnement de stimulation ou de motiver à faire la recherché [4].

L'Etat est une personne morale publique dont le devoir est d'assurer le bien-être de la communauté nationale. Auparavant, son rôle était limité aux tâches régaliennes.
Mais aujourd'hui, l'Etat a connu une évolution. L'Etat moderne n'est plus seulement un Etat gendarme, mais aussi un Etat interventionniste. C'est-à-dire que l'Etat joue un rôle dans tous les domaines de la vie de la communauté dans le souci de promouvoir le bien-être social et le développement économique pour s'assurer un équilibre budgétaire entre les entités administratives décentralisées.

Cependant, chaque année les entités élaborent, exécutent et éventuellement contrôlent leur budget dont dépend le fonctionnement de leurs administrations respectives, conformément à la constitution de février 2006, tel que modifié et complété à ce jour, ayant institué une nouvelle politique de décentralisation qui accorde des larges pouvoirs aux entités administratives décentralisées qui a pour but de faire des entités territoriales décentralisées des véritables centre d'impulsion.

Certes, l'article 3 de la constitution stipule que les provinces et les entités territoriales de la République Démocratique du Congo jouissent de la libre administration et de l'autonomie de gestion de leurs ressources économiques, humaines, financières et techniques.

D'après la constitution, les entités administratives sont habilitées à retirer à la source $40 \%$ des recettes produites par ces dernières, les $60 \%$ restant devant être envoyer au gouvernement central.

Ainsi, pour assurer un équilibre fiscal, le fonctionnement de ces entités territoriales ainsi que leurs rapports avec l'Etat et les provinces sont fixées par la loi organique dans son article 149 chapitre II sur les finances, cédant quelques impôts et taxes aux entités administratives décentralisées. Ceux-ci feront l'objet des questions que nous tenterons de répondre tout au long de notre étude, notamment:

- La retenue à la source des recettes par les ETD s'effectue-t-elle selon l'esprit de la loi à la matière pour leur développement?

- Dans le cas contraires, quelles en sont les conséquences et pistes de solutions?

Toutes ces préoccupations faisant l'objet de notre étude ne manqueront pas de susciter ou d'attirer l'attention de plus d'uns, d'où l'intérêt de cette étude.

\section{HYPOTHESE}

Une hypothèse est une série de réponses qui permettent de prédire la vérité scientifique. La vois semblance au regard des questions soulevées par la problématique et la recherche à vérifier le bien ou le mal fondé [5]. 
Prenant comme repère cette définition et nous penchant sur les questions formulées ci-haut, nos réponses provisoires quant à ce se présentent comme suit:

Depuis son instauration, loi sur la retenue à la source de $40 \%$ des recettes à caractère national par les provinces souffrirait d'une violation systématique, surtout sur le point abordant la retenue à la source par les ETD de $40 \%$. Cette retenue ne s'effectuerait pas, partout dans le pays. Une situation qui s'expliquerait par une absence brillante de volonté politique du pouvoir central.

Les conséquences seraient énormes et d'ailleurs, trop visibles. Les ETD se trouvent dans une dépendance économique au pouvoir central, chose qui, conséquemment, freine leur développement. Pour y remédier, le pouvoir central devrait veiller avec bonne foi à la transparence dans la rétrocession des recettes dues aux ETD.

\section{OBJECTIFS DE L'ETUDE}

Ce travail vise, de manière générale, à analyser la praticabilité de la loi quant à la retenue à la source par les ETD. Nous essayerons de voir son respect ou non. Ainsi, les autorités politiques du pays à tous les niveaux ainsi que toute autre personne intéressée par cette question trouveront un intérêt particulier aux résultats qui, à notre humble avis, proposeront certaines pistes des solutions plausibles.

\section{CHOIX ET INTERET DU SUJET}

L'aspect financier de la décentralisation, demeure un sujet d'actualité en RDC étant donné que ce processus n'a jamais abouti de manière satisfaisante en dépit de multiplicité des lois.

La présente recherche se justifie par l'actualité même du sujet abordé, car la décentralisation dans sa version « retenue à la source de $40 \%$ et ou encore, la rétrocession » est une question épineuse et un défi à relever par l'Etat congolais s'il veut vraiment décoller et se retrouver parmi les pays émergeants dans un temps raisonnable.

Sur le plan scientifique, la présente étude servira pour les chercheurs sans omettre l'Etat congolais comme un manuel globalisant un certain nombre de réponses aux questions bloquant la mise en œuvre de la loi abordant la retenue à la source.

\section{CADRE METHODOLOGIQUE}

Selon PINTO Et GRAWITZ [6], la méthode est un ensemble d'opération intellectuelle par lesquelles une discipline cherche à atteindre les vérités qu'elle poursuit, les démontre et les vérifie.
Pour vérifier et appréhender la question de notre présente étude, il nous semble utile de recourir à une méthode spécifique. Nous allons dans ce travail, utilisé la méthode juridique, celle-ci consiste selon PINTO R., et GRAWITZ M., [7] à interpréter un texte légal selon les normes de critique scientifique bien structuré.

Cette méthode va nous aider pleinement à véhiculer aux tours des textes abordant la rétrocession, la retenue à la source ainsi que la décentralisation.

Le fait que nous serons dans l'obligation de palper du doigt les prescrits des divers documents sur la matière à analyser dans le présent travail, c'est pourquoi, la technique documentaire est sans doute pour nous, plus pratique, explicite et appropriée dans la réalisation de notre modeste travail. Elle consiste à analyser des documents tels que les archives, les documents officiels écrits, sonores ou audio-visuels, ..., de manière objective avec pour but de les interpreter [8].

\section{DELIMITATION DE L'ETUDE}

Aborder tous les aspects d'un sujet aussi complexe comme celui-ci, est littéralement impossible. Il est donc nécessaire de délimiter notre sujet dans le temps et dans l'espace pour nous permettre de réaliser un travail consistant.

Dans le temps notre étude s'étend de 2016 à 2018, la période à laquelle, les districts sont devenus provinces. Et dans l'espace, l'Ituri est notre champ d'application.

\section{PLAN SOMMAIRE}

Dans cette étude, nous allons aborder les points suivant: les modalités de répartition des recettes entre le pouvoir central et les provinces ainsi que les ETD, du mécanisme de transfert financier : rétrocession et la caisse de péréquation et enfin nous analyserons la retenue à la source de $40 \%$ : théorie ou pratique s'agissant de la province de l'Ituri.

\section{MODALITES DE REPARTITION DES RECETTES ENTRE LE POUVOIR CENTRAL ET LES PROVINCES AINSI QUE LES ETD \\ Des ressources provenant des recettes à caractère national}

En prenant pour appui l'article 54 de la loi $n^{\circ} 08-012 d u 31$ juillet 2008 portant principes fondamentaux relatifs à la libre administration des provinces, la part des recettes à caractère national allouées aux provinces est établie à $40 \%$. Elle est retenue, en principe à la source.

Il est précisé que la retenue à la source s'effectue par le versement automatique de $40 \%$ dans le compte de la province et de $60 \%$ dans le compte général 
du trésor public. Il est également nécessaire de préciser que le mécanisme dont il est ici question est exécuté, sans commentaire par la banque centrale du Congo, et ce, conformément à la loi des finances.

De toute évidence, un questionnement pourrait surgir quant aux recettes qui peuvent être considérées comme faisant parties ou étant des recettes à caractère national. Pour en savoir plus, l'article 55 de la loi précitée est éloquent quant à ce. Ainsi donc, sont considérés comme recettes à caractère national:

- Les recettes administratives, judiciaires domaniales et de participation ;

- Les recettes de douanes et accises ;

- Les recettes provenant des impôts recouvrés sur les grandes entreprises des pétrolières productrices ainsi que les autres impôts pouvant être perçus à leur lieu de réalisation.

Partant de cet article 55 cité ci-dessus, l'on comprend aisément que les recettes à caractère national sont recouvrées par les régies financières que sont la DGRAD, la DGDA, DGE et la DGI. Ces recettes sont donc de la compétence du pouvoir central. Toutefois, elles sont répartissables entre trois catégories de bénéficiaires: le pouvoir central, la province et les collectivités publiques locales.
En outre, il serait illogique, estimons-nous, de ne pas survoler ne serait-ce qu'en quelques mots les charges publiques de l'Etat, tout en relevant des recettes et dépenses de l'Etat. Dans cette optique, il serait souhaitable, compte tenu du principe budgétaire de la légalité des recettes et des dépenses de l'Etat, de s'orienter dans la nomenclature à jour en RDC, parlant des recettes et une autre des dépenses publiques de l'Etat.

Le budget de l'Etat comprend le budget du pouvoir central et le budget de la province. Il est arrêté chaque année par une loi. (Art 44 loi 2008, portant principes fondamentaux sur la libre administration des provinces).

Il s'est avéré que l'ordonnance-loi n $13 / 001 \mathrm{du}$ 23 février 2013 reprend les recettes cédées par l'Etat aux provinces et aux ETD tirées de la nomenclature des recettes sus évoquées. Nous devons reconnaitre de tout état de chose, que toute collectivité publique, que ce soit le pouvoir central, les provinces et les ETD doivent élaborer leur budget en tenant compte des libellés budgétaires contenus dans ces deux nomenclatures des recettes que nous représentons, pour une compréhension aisée, dans le tableau qui suit.

Tableau-1: Nouvelles nomenclatures des recettes et des dépenses en RDC

\begin{tabular}{|c|c|}
\hline RECETTES & DEPENSES \\
\hline I. Recettes courantes & I. Dépenses courants \\
\hline I.1. Recettes à caractère national & Titre I: Dette publique en capital \\
\hline I.2. Recette d'intérêt commun & Titre II : Frais financiers \\
\hline II.3. Recettes spécifiques & Titre III : Dépenses du personnel \\
\hline & Titre IV : Biens et matériels \\
\hline II. Recette en capital & Titre V : Dépenses de prestation \\
\hline II.1. Produit de cession d'actif & Titre VI : Transferts et interventions \\
\hline $\begin{array}{l}\text { III. Recettes exceptionnelles } \\
\text { III.1. Dons et legs } \\
\text { III.2. Subventions éventuelles }\end{array}$ & $\begin{array}{l}\text { II. Dépenses en capital } \\
\text { Titre VII : Equipements } \\
\text { Titre VIII : Construction, réfection, réhabilitations, additions } \\
\text { d'ouvrages et édifices, acquisitions immobilière. }\end{array}$ \\
\hline $\begin{array}{l}\text { IV. Ressources extérieures } \\
\text { IV.1. Dons et legs extérieurs courants } \\
\text { IV.2. Dons et legs extérieurs projets } \\
\text { d'investissement }\end{array}$ & $\begin{array}{l}\text { III. Dépenses des prêts et avance } \\
\text { Titre IX : Prêts et avances }\end{array}$ \\
\hline
\end{tabular}

Otemikongo, M [9, 10], Table-5, notes des cours de G2 Droit, UNIBU (2013-2014)

Nous nous réservons présentement le droit de faire un quelconque commentaire concernant le tableau ci-haut; toutefois, dans les lignes qui suivent, nous essayerons d'y revenir en vue de placer notre point de vue quant à ce.

\section{Ressources Financières De La Province}

La loi $n^{\circ} 08-12$ du 31 Juillet 2008 portant principes fondamentaux relatifs à la libre administration des provinces distingue, d'une part, les ressources de la province (art 48) et d'autre part, les ressources provenant des recettes à caractère national (Art 55).

Il y a lieu également de dire que les budgets des ETD sont intégrés, en dépenses et en recettes, dans le budget de la province conformément aux dispositions de la loi de finance (Art 45 de la loi $\mathrm{n}^{\circ} 08-012 \mathrm{du} 31$ juillet 2008). 
Abordant présentement les notions et énumérations des ressources propres de la province, celles-ci comprennent les impôts, les taxes, les droits provinciaux et locaux ainsi que les recettes de participation (art 48 de la loi précitée).

Nous pouvons donc dire que c'est la province qui établit le mécanisme de leur recouvrement dans le respect des procédures fixées par la législation à la matière et, en respectant les normes y afférentes.

Les taxes, les droits provinciaux et locaux comprennent notamment: les taxes d'intérêt commun, les taxes spécifiques à chaque province et à chaque entité et les recettes administratives rattachées aux actes générateurs dont la décision relève de la compétence des provinces. (Art 40 de la loi $n^{\circ} 08-012$ du 31 juillet 2008).

Néanmoins, les taxes d'intérêt commun, comprennent entre autres: la taxe spéciale de circulation routière ; la taxe annuelle pour la délivrance de la patente; diverses taxes de consommation sur la bière, l'alcool et spiritueux ainsi que le tabac ; la taxe de superficie sur les concessions forestières; la taxe de superficie sur les concessions minières; la taxe sur les ventes des matières précieuses de production artisanale ; toutes les autres taxes instituées par le pouvoir central et revenant en tout ou en partie aux provinces en vertu de la loi (Art 50 de la loi $\mathrm{n}^{\circ}$ 08-012 du 31 juillet 2008).

Il sied de rappeler que la clé de répartition du produit des taxes d'intérêt commun entre les provinces et les entités territoriales décentralisées (ETD) est fixée par la législation qui institue les dites taxes, après avis de la conférence des gouverneurs de province [11].

En dehors des éléments précités, la province a également droit: aux recettes provenant des taxes spécifiques prélevées sur les matières locales non imposées par le pouvoir central. Ces taxes spécifiques sont soit rémunératoires, soit fiscales conformément à la législation sur la nomenclature des taxes et droits provinciaux. Les règles de perception des taxes spécifiques sont fixées, après avis de la conférence des gouverneurs de province, par la loi fixant la nomenclature des recettes locales (Art 51). aux recettes administratives rattachées aux actes générateurs dont la décision relève de sa compétence; aux recettes de participation comprenant les bénéfices ou les revenus de sa participation au capital des entreprises et association sans but lucrative [11]

\section{Ressources Financières Des ETD}

Nous l'avions juste énoncé dans le premier chapitre de ce travail que les ressources financières des ETD sont organisées par les articles 171 à 175 de la
Constitution du 18 février 2006, 104 à 119 de la loi organique $\mathrm{n}^{\circ} 08 / 016$ du 7 octobre 2008 et 147,148 et 149 de la loi financière $n^{\circ} 11 / 011$ du 13 juillet 2011 . De cela, peut-on dégager: les recettes courantes; les recettes en capital ainsi que les recettes exceptionnelles.

\section{RETENUE A LA SOURCE DE 40\%: THEORIE OU PRATIQUE \\ Théorie}

Nous ne le dirons pas assez, au-delà des aspects et facteurs analysés profondément dans les points précédents de la présente étude, cela n'empêche aucunement de revenir sommairement sur principalement, le partage des compétences et ressources entre l'Etat et les Provinces, lequel déjà bien défini dans la constitution du 16 février 2006 tel que modifié à ce jour.

Parlant des compétences exclusives du pouvoir central et les Provinces; dans le cadre de leurs compétences exclusives, les deux niveaux de pouvoir ont leurs prérogatives bien définies. Pour ce faire, aucun niveau ne peut interférer dans le fonctionnement de l'autre, mais tout en maintenant la collaboration entre elles. Par ailleurs, toute révision constitutionnelle ayant pour objet ou pour effet de réduire les prérogatives de province est interdite. Ce qui confirme la forme régionale voire fédérales de l'Etat congolais.

Ainsi donc, nous sommes face à un régionalisme qui suppose que les provinces ont la libre disposition pour la solution des problèmes qui se posent à elles dans le cadre de leurs compétences? Ainsi, chaque Province trouvera des solutions spécifiques à ses problèmes. Ce qui peut supposer que les mêmes problèmes qui se posent aux provinces pourraient être solutionnés de manière différenciée. (Art 201, 202 et 204 Constitution de 2006 telle que modifiée a ce jour)

Alors que, le partage des recettes et l'autonomie budgétaire des provinces (art 171 et 175), des dispositions constitutionnelles garantissent l'autonomie financière des provinces.

L'article 171 stipule clairement que «les finances du pouvoir central et celles des provinces sont distinctes ». Ainsi, les provinces fonctionnent sur base d'un budget qui leur est propre et non intégré dans le cadre du budget central. Néanmoins, la loi de programmation budgétaire doit intégrer le budget de provinces comme le stipule l'article 175 alinéa 1 de la constitution.

Les provinces élaborent et gèrent librement leur budget selon leurs priorités. Toutefois, cette autonomie s'exerce dans le cadre des compétences fixées par la constitution soit les compétences exclusives ou concurrentes. 
Menga Yaosuwa \& Imani Gaya., Sch Bull, Nov 2019; 5(11): 624-634

Par ailleurs, il est institué à l'article 175 de la Constitution un principe de partage des recettes entre l'État et les provinces. Ainsi, l'article 175 précise que: « La part des recettes àcaractère national allouées aux provinces est établie à $40 \%$. Elle est retenue à la source. » La Constitution établit donc le principe de péréquation quant à la distribution des recettes à Caractère national entre l'État et les Provinces. Cet article prévoit de plus que la part des recettes à être reversée aux Provinces sera retenue à la source. Cette disposition garantit donc que le budget des Provinces sera alimenté au fur et à mesure de l'entrée des recettes nationales.

Des mécanismes de mise en application de ces dispositions doivent encore être définis. L'épineuse question de savoir ce que représentent les recettes nationales est clarifié par la loi du 10 juillet 2008 portant principes fondamentaux relatifs à la libre administration des Provinces et spécifiquement à son article 55 «Sont à caractère national, les recettes administratives, judiciaires, domaniales et de participation, les recettes de douanes et accises, les recettes provenant des impôts recouvrés sur les grandes entreprises, des producteurs pétroliers ainsi que les autres impôts pouvant être perçus à leur lieu de réalisation ».

Néanmoins plusieurs questions restent en suspens notamment, des recettes peuvent être perçues ailleurs que sur le lieu de production ou de consommation des produits. Un des exemples les plus illustratifs est le port de Matadi où l'on perçoit des recettes des produits importés ou exportés qui ne sont pas consommés dans la Province du Bas-Congo. Des infrastructures financées par l'Etat central participeront à l'augmentation des recettes dans certaines provinces alors que toutes les provinces ont contribué à leur construction. De ce fait, comment se répartiront les recettes tirées de ces infrastructures? [12].

\section{Pratique}

Dans ce dernier point de notre travail considéré comme crucial, nous allons revenir sommairement sur ce que prévoient les lois congolaises en termes de retenue à la source et/ou rétrocession et ce qui se passe effectivement sur le terrain pour en déduire un décalage s'il en aura.

Comme tous les éléments indispensable ont été déjà analysé dans les points différents de la présente étude, ce qui importe pour nous présentement, est de donner une image nette de l'application d'une part, de la retenue à la source de $40 \%$ des recettes à caractère national par l'Ituri et, d'autre part si nécessité il y en a, jauger la mise en œuvre de la rétrocession de $40 \%$ des recettes dont il est question ici.

Ainsi donc, pour arriver à notre fin, nous avons collectionné des données chiffrées que nous allons condenser dans le tableau ci-dessous avant d'en décortiquer la quintessence.

Tableau-2

\begin{tabular}{|l|l|l|l|l|l|}
\hline $\mathbf{N}^{\circ}$ & ANNEES & $\begin{array}{l}\text { BUDGET } \\
\text { GENERAL DE LA } \\
\text { PROVINCE DE } \\
\text { L'ITURI }\end{array}$ & $\begin{array}{l}\text { PART DES RECETTES A } \\
\text { CARACTERE } \\
\text { NATIONAL A ALLOUER } \\
\text { AUX PROVINCES }\end{array}$ & $\begin{array}{l}\text { Ressources } \\
\text { internes de } \\
\text { province }\end{array}$ & $\begin{array}{l}\text { Recettes à caractère } \\
\text { national } \\
\text { effectivement alloué } \\
\text { à l'Ituri }\end{array}$ \\
\hline 01 & $\mathbf{2 0 1 6}$ & $11.315 .988 .284,14$ & $5.162 .658 .957,70$ & $11.315 .988 .284,14$ & $\mathbf{4 . 6 6 4 . 5 2 0 . 1 3 9 , 3 5}$ \\
\hline 02 & $\mathbf{2 0 1 7}$ & $68.501 .314 .086,97$ & $42.364 .214 .701,74$ & $50.495 .103 .135,68$ & $\mathbf{2 . 9 4 2 . 8 2 0 . 7 5 4}$ \\
\hline 03 & $\mathbf{2 0 1 8}$ & $113.345 .039 .040,76$ & $47.995 .895 .625,00$ & $64.323 .325 .191,75$ & $\mathbf{2 . 9 4 2 . 8 2 0 . 7 5 4}$ \\
\hline
\end{tabular}

Source: confectionné par nous-mêmes tenant compte des données reçues du département finance du gouvernorat de la province de l'Ituri.

Tableau-3: Prévision des recettes du gouvernement provinciale de 2016 à 2018

\begin{tabular}{|l|r|l|r|}
\hline Eléments de prévision de recette & \multicolumn{1}{|l|}{$\mathbf{2 0 1 6}$} & $\mathbf{2 0 1 7}$ & $\mathbf{2 0 1 8}$ \\
\hline Budget Général & 11315988284.15 & 68501314086.97 & 113345039040.76 \\
\hline Part de recette nationale alloué à la province & 5162658957.70 & 42364214701.74 & 74985895625 \\
\hline
\end{tabular}

Source: Service Budget de la province

Les recettes de Budget provinciale ont été prévues $11315988284.15,68501314086.97$ et 113345039040.76 respectivement pour les années 2016, 2017 et 2018 et la part de recette nationale alloué à la province ont été prévues 5162658957.70 , 42364214701.74 et 74985895625 respectivement pour les mêmes années.

Tableau-4: Réalisation des recettes du gouvernement provinciale de 2016 à 2018

\begin{tabular}{|l|r|r|r|}
\hline Eléments de réalisation de recette & \multicolumn{1}{|l|}{$\mathbf{2 0 1 6}$} & $\mathbf{2 0 1 7}$ & $\mathbf{2 0 1 8}$ \\
\hline Ressource interne de la province & 11315988.14 & 50495103135.68 & 64323325191.75 \\
\hline Recette à caractère nationale alloué à l'Ituri & 4664520139.35 & 2942820754 & 2942820754 \\
\hline
\end{tabular}

Source: Service Budget de la province 
Les recettes due aux ressources internes de Budget provinciale ont été réalisées pour 11315 988.14, 50495103135.68 et 64323325191.75 respectivement pour les années 2016, 2017 et 2018 et la part de recette nationale alloué à la province ont été réalisées sont de 5162658 957.70, 42364214701.74 et 74985895625 respectivement pour les mêmes années.

La variation des prévisions $=100-\left(\frac{\text { Prévision de l'année } n}{\text { Prévision del'année } n+1} \times 100\right)$

La variation des réalisations $=100-\left(\frac{\text { Réalisation de l'année } n}{\text { Réalisation de l'année } n+1} \times 100\right)$

Tableau-5: Tableau des variations des recettes internes de 2016 à 2018

\begin{tabular}{|l|r|r|r|r|}
\hline Années & Prévisions & Variations & Réalisation & Variations \\
\hline 2016 & 11315988284.15 & - & 11315988.14 & - \\
\hline 2017 & 68501314086.97 & 83.48 & 50495103135.68 & 99.978 \\
\hline 2018 & 113345039040.76 & 39.56 & 64323325191.75 & 21.50 \\
\hline
\end{tabular}

Source: Fait par nous même à partir des tableaux 1 et 2 .

Dans ce tableau, il ressort que les prévisions des recettes internes ont connu une variation $65.13 \%$ de 2016 à 2017. de 2017 à 2018, ces prévisions ont connu

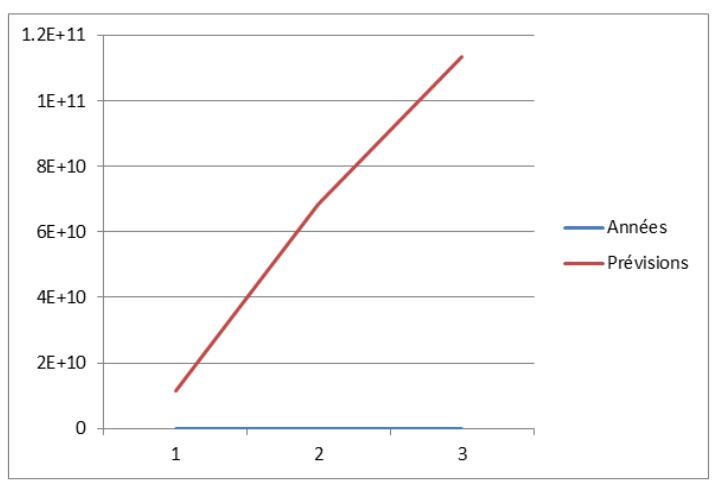

Graphique-1: évolution des prévisions des recettes internes de 2016 à 2018 une variation de 39,57 . Les réalisations ont connu une variation de $99.98 \%$ de 2016 à 2017 et une variation de $21,502 \%$ de 2017 à 2018.

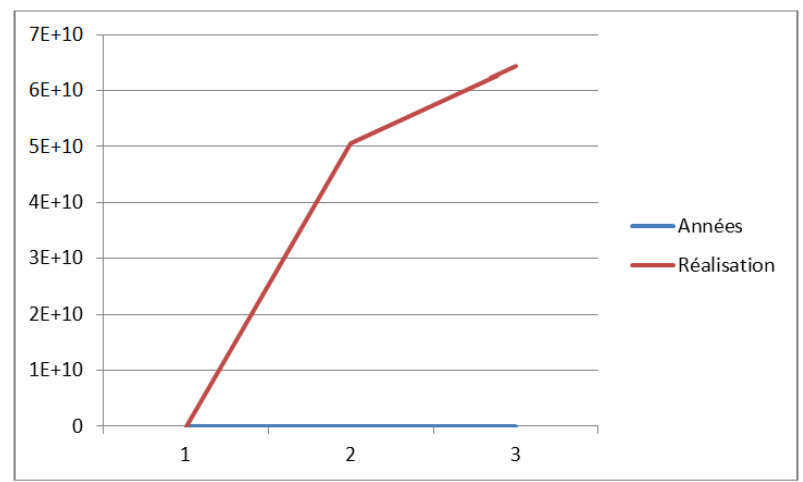

Graphique-2: Évolution des Réalisations des recettes internes des 2016 à 2018

Tableau -6: Tableau des variations des recettes nationale alloué à la province de 2016 à 2018

\begin{tabular}{|l|r|r|r|r|}
\hline Années & \multicolumn{1}{|c|}{ Prévisions } & Variations & Réalisation & Variations \\
\hline 2016 & 5162658957.70 & - & 11315988.14 & - \\
\hline 2017 & 42364214701.74 & 87.81 & 50495103135.68 & 99.98 \\
\hline 2018 & 74985895625 & 43.50 & 64323325191.75 & 21.50 \\
\hline
\end{tabular}

Source: Fait par nous même à partir des tableaux 1 et 2 .

De ce table, il est a noter que les prévisions des recettes internes ont connu une variation $65.13 \%$ de 2016 à 2017. de 2017 à 2018, ces prévisions ont connu une variation de 39,57. Les réalisations ont connu une variation de $99.98 \%$ de 2016 à 2017 et une variation de $21,502 \%$ de 2017 à 2018. 


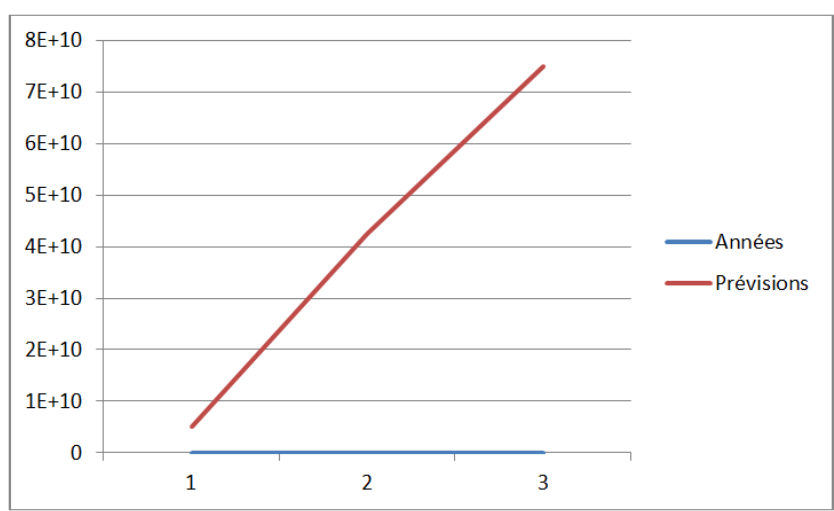

Graphique-3: Évolution des prévisions recettes nationale alloué à la province

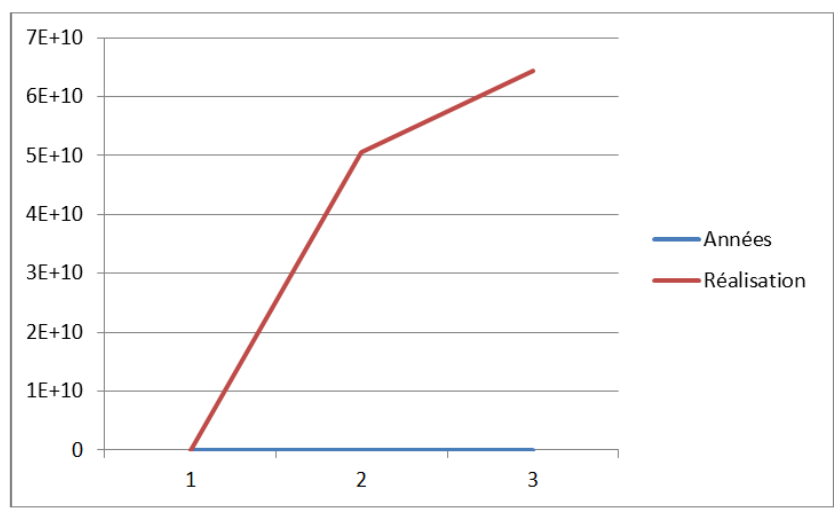

Graphique-4: Évolution des Réalisation de recettes nationale alloué a la province

Tableau-7: Tableau des écarts des recettes nationales prévues et réalisées alloué à la province 2016 à 2018

\begin{tabular}{|c|l|l|l|c|c|}
\hline \multirow{2}{*}{ Années } & \multirow{2}{*}{ Prévisions } & \multirow{2}{*}{ Réalisations } & \multicolumn{2}{|l|}{ Ecarts } & \multirow{2}{*}{ Taux de réalisation } \\
\cline { 4 - 5 } & & & Favorable & Défavorables & \\
\hline 2016 & $5.162 .658 .957,70$ & $4.664 .520 .139,35$ & - & $498.138 .818,35$ & $90,35 \%$ \\
\hline 2017 & $42.364 .214 .701,74$ & 2.942 .820 .754 & - & $39.421 .383 .947,74$ & $6,95 \%$ \\
\hline 2018 & $47.995 .895 .625,00$ & 2.942 .820 .754 & - & $45.053 .074 .871,00$ & $6,13 \%$ \\
\hline
\end{tabular}

Source: Fait par nous même à partir des tableaux 3 et 4 .

De ce tableau, il ressort les recettes nationales prévues en 2016 ont été réalisées à 90,35\%. En 2017, elles sont été réalisées à $6,95 \%$ et en 2018 , elles ont été réalisées à $6,13 \%$. Les écarts entre les prévisions et les réalisations se sont élèves à 498138 818,35 en 2016, 39421383947,74 en 2017 et 45053074871.00 en 2018. L'écart a été défavorable pour ces trois années.

Avant de procéder à l'interprétation approfondie de ce muni tableau ci-dessus, il nous semble indispensable de préciser que la colonne réservée au budget général de la province de l'Ituri, correspond à la prévision budgétaire annuel de ladite province.

Concernant la part des recettes à caractères national à allouer aux provinces, c'est donc le montant que devrait bénéficier la province de l'Ituri tenant compte de chacune des années. Les ressources internes de la province, représente la capacité de mobilisation financière interne de l'Ituri alors que les recettes à caractère national effectivement alloué à l'Ituri, correspond exactement au montant reçu par l'Ituri du pouvoir central.

Aussi, faut-il ajouter qu'en principe, dans la part des recettes à caractère national alloués à la province, sont inclus, les transferts en faveur de l'Assemblée Provinciale, le transfert au titre de fonctionnement reçu pour la province et les ETD et une partie des investissements qui n'arrive malheureusement jamais.

Concernant l'année 2016, une année qui coïncide avec l'avènement de la politique nationale de la mise en œuvre effective de décentralisation administrative en RDC, où les anciens districts devraient pleinement être appelés des provinces et les devenir effectivement, l'Ituri comme d'ailleurs d'autres districts n'a pas eu un budget nettement programmé. Aussi, le 11.315.988.284,14 ne représente qu'un budget trop estimatif et non détaillé. Et ceci plus soutenu par la migration de la DGRPO à la DGRPI pour le compte 
propre de l'Ituri. (Sources: service de Finances de province Ituri).

Avant de porter une analyse particulière par rapport à la rétrocession de $40 \%$, nous trouvons utiles de rappeler que, suivant le tableau sus indiqué, le budget de la province de l'Ituri est plutôt beaucoup plus évolutif. De 11.315.988.284,14 en 2016, l'Ituri est passé à 68.501.314.086,97 au cours de l'an 2017 pour atteindre 113.345.039.040,76 en 2018. Ce qui montre une bonne progression vers la maximisation des recettes par les services y afférents en Ituri. D'où en termes de pourcentage, l'on dira que le budget de l'Ituri a progressé d'environ 10\% de 2016 à 2018.

Parlant de la rétrocession de $40 \%$ des recettes à caractère national aux provinces, trois commentaires suivant doivent être fait:

En 2016, sur les 5.162.658.957,70 FC (Franc Congolais, Cinq milliards cent soixante deux mille, six cent cinquante-huit mille neuf cent cinquante-sept centimes septante) que l'Ituri s'attendait et/ou devrait recevoir, seulement 4.664.520.139,35 FC (Francs Congolais quatre milliards six cent soixante-quatre millions cinq cent vingt mille cent trente-neuf centimes trente-cinq) qu'elle a eu effectivement à recevoir de la part de pouvoir central (Kinshasa). Soit un écart d'environs 498.138.818,35 FC (Franc Congolais, quatre millions cent trante huit mille huit cent dix-huit centimes trante-cinq) ;

Par contre, en 2017 sur les 42.364.214.701,74 FC (Franc Congolais, quarante deux milliards trois cent soixante quatre millions deux cent quatorze mille sept cent et un centimes septante quatre) auxquels s'attendait l'Ituri, seulement 2.942.820.754 FC (Franc Congolais, deux milliards neuf cent quarante deux millions huit cent vingt milles sept cent cinquante quatre) qu'a alloué le pouvoir central dans la rubrique des recettes à caractère national alloués aux provinces.

Ce qui représente un écart de plus au moins 39.421.393.947,74 FC (Trante neuf millions quatre cent vingt et un mille neuf cent quarante centimes septante quatre) ;

Enfin, en 2018 comme en 2017, seulement 2.942.820.754 FC (Franc Congolais, deux milliards neuf cent quarante deux millions huit cent vingt milles sept cent cinquante quatre) ont été alloués à l'Ituri sur les 47.995.895.625 FC (quarante sept milliards neuf cent nonante cinq millions huit cent nonante cinq mille six cent vingt-cinq) initialement attendus par la province de l'Ituri. Un écart net de 45.053.074.871 FC (quarante cinq milliards millions cinquante trois millions septante quatre mille huit cent septante et un).

Des explications ci-haut, nous relevons que la rétrocession semble diminuer année après année. En termes de pourcentages, voici ce que ça peut représenter:

En 2016, l'Ituri n'est parvenu qu'à recevoir 90,1\% du montant initialement attendus; En 2017 par contre, une chute libre a été observé soit seulement environs $\mathbf{7 \%}$ du montant attendus par la province;

Et en 2018, une diminution encore d'un 1\% pour donner $6 \%$ en ce que s'attendait la province de l'Ituri.

De ce qui précède, nous relevons que la province de l'Ituri n'a bénéficié pleinement de la rétrocession qu'au cours de l'année 2016 bien que la totalité du montant attendu n'a pas été envoyé par Kinshasa.

Mais aussi, tel que nous l'avons remarqué, il y a une brillance absence d'une base standard pouvant permettre à calculer sans faille la totalité de $40 \%$ que devrait recevoir chacune des nouvelles provinces. D'où, le pouvoir central n'envoie que des montants forfaitaires aux provinces, sans pour autant tenir comptes de leurs capacités de mobilisations des recettes d'une part, et d'autre part, le budget annuel.

Par ailleurs, tout en soutenant l'autonomie budgétaire de chaque province, nous estimons que, l'article 175 de la constitution est loin d'être mise en application. Pour rappel, il précise que : «La part des recettes àcaractère national allouées aux provinces est établie à $40 \%$. Elle est retenue à la source ».

Donc, entre la retenue à la source et la rétrocession de $40 \%$, c'est plutôt la rétrocession forfaitaire qui est d'application pourtant, privé de tout fondement juridique le soutenant. Ce qui pour nous a comme conséquence, le freinage de relance économique des nouvelles provinces de la RDC dont l'Ituri, qui ne reçoivent pas suffisamment des moyens du pouvoir central pour entamer le fameux concept de «décollage». Cette rétrocession n'est donc pas en totalité effective vu son caractère forfaitaire et insignifiant au vu de toutes les charges qu'on les provinces.

$\mathrm{Au}$ stade actuel, nous sommes persuadés d'avoir suffisamment évoqué chacun des points essentiels de notre étude.

Rappelons par contre que la composition, l'organisation ainsi que le fonctionnement des ETD et leurs rapports avec l'Etat et les provinces sont régies par la loi organique $n^{\circ} 08-016$ du 07 octobre 2008 qui stipule en son article 100 que: «les finances des Entités Territoriales Décentralisées sont distinctes de celles des provinces ». 
Cette loi organique distingue également en ce qui concerne les ressources propres et, d'autre part, les ressources provenant des recettes à caractère national allouées aux provinces, les ressources de la caisse nationale de la péréquation ainsi que les ressources exceptionnelles.

De ce qui précède, les ressources propres des entités territoriales décentralisées comprennent, en vertu de l'article 108 de la loi précitée:

L'impôt personnel minimum; qui est perçu au profit exclusif des communes, des secteurs ou des chefferies. Il est établit et recouvré conformément à la loi. « la clé de répartition du produit des taxes d'intérêt commun entre les Entités Territoriales Décentralisées est fixée par la législation qui institue les dites taxes, après avis de la conférence des gouverneurs de province ».

Les recettes de participation; elles comprennent les bénéfices ou revenus de leur participation au capital des entreprises publiques, des sociétés d'économie mixte et des associations momentanées à but économique.

Les taxes et droit locaux; comprennent les taxes d'intérêt commun, taxe spéciale de circulation routière, taxe annuelle sur la patente, taxes diverses de consommation sur la bière et le tabac, la taxe de superficie sur les concessions forestières et minières, taxes sur les ventes des matières précieuses de production artisanale et toutes autres taxes instituées par le pouvoir central et revenant en tout ou en parti à l'entité territoriale décentralisée en vertu de la loi (Art 112).

Les taxes spécifiques à chaque entité territoriales décentralisées sont des taxes prélevées sur les matières locales non imposées par le pouvoir central. Elles sont soit remontoirs, soit fiscales conformément à la législation sur la nomenclature des taxes et droits provinciaux et locaux.

«Les règles de perception des taxes spécifiques sont fixées, après avis de la conférence des gouverneurs, par la loi fixant la nomenclature des recettes locales $\gg$.

Les recettes administratives rattachées aux actes générateurs dont la décision relève de la compétence de l'Entité Territoriale Décentralisée.

Comme les provinces, Les Entités Territoriales Décentralisées ont droit à $40 \%$ de la part des recettes à caractère national allouées aux provinces. " $L a$ répartition des ressources entre les Entités Territoriales Décentralisées est fonction des critères de capacité de production, de la superficie et de la population. L'Édit en détermine le mécanisme de répartition ».

Concernant des ressources de la caisse nationale de péréquation (article 117) et des ressources exceptionnelles: une Entité Territoriale Décentralisée peut recourir aux emprunts intérieurs pour financer ses investissements ; elle peut également bénéficier des dons et legs dans les conditions définies par la loi. (KAPYA KABESA., 2014 :30).

Revenant sur le tableau repris dans le point (II.1.1) l'on peut retenir que les ETD ont inéluctablement besoin de budgets conséquents au regard des charges publiques auxquelles elles doivent faire face pour ainsi contribuer efficacement au développement de l'Etat congolais à partir de la base. Ces charges sont contenues dans la nomenclature des dépenses publiques de l'Etat, édition 2004.

\section{CONCLUSION}

La décentralisation telle qu'instituée en République Démocratique du Congo ne doit pas rester un slogan. Il faudrait qu'elle devienne effective et réelle pour servir au développement du pays tout entier à partir du développement de l'ensemble des entités de base.

En analysant l'ordonnance loi n'82/006 du 25 février 1982 telle que modifiée par la loi du 20 décembre 1995, la loi du 2 juillet 1998 et constitution du 18 février 2006 votée par referendum en 2005 instituant une nouvelle politique de décentralisation qui accorde des larges pouvoirs aux entités décentralisées, nous pouvons en effet estimer que la réussite des objectifs des entités administratives décentralisée passe par l'aménagement de celles-ci à tous les niveaux afin d'activer la mission première qu'elles se sont assignées pour donner la meilleure d'elles-mêmes.

Ces entités décentralisées sont des entités de base et ne peuvent être efficaces viables que si elles ont à leur disposition, en plus des hommes agents de développement, des ressources suffisantes.

C'est ainsi donc que nous sommes parvenues à conclure qu'entre la retenue à la source et la rétrocession de $40 \%$, c'est plutôt la rétrocession forfaitaire qui est d'application pourtant, privé de tout fondement juridique le soutenant.

Mais aussi, tel que nous l'avons remarqué, il y a une brillante absence d'une base standard pouvant permettre à calculer sans faille la totalité de $40 \%$ que devrait recevoir chacune des nouvelles provinces.

Par ailleurs, tout en soutenant l'autonomie budgétaire de chaque province, nous estimons que, l'article 175 de la constitution est loin d'être mise en 
Menga Yaosuwa \& Imani Gaya., Sch Bull, Nov 2019; 5(11): 624-634

application. Pour rappel, il précise que: «La part des recettes àcaractère national allouées aux provinces est établie à $40 \%$. Elle est retenue à la source. \C Ce qui pour nous a comme conséquence, le freinage de relance économique des nouvelles Provinces de la RDC dont l'Ituri, qui ne reçoivent pas suffisamment des moyens du Pouvoir Central pour entamer le fameux concept de «écollage ».

\section{BIBLIOGRAPHIE \\ TEXTES LEGAUX ET REGLEMENTAIRES}

- Constitution de la République Démocratique du Congo, in Jo-RDC, $\mathrm{N}^{\circ}$ spécial, $47^{\text {ème }}$ année, CEDI, Kinshasa, 18 février 2006 telle que modifiée à ce jour.

- Loi $\mathrm{n}^{\circ} 08 / 12 \mathrm{du} 31$ juillet 2008 portant principes fondamentaux pour la libre administration des provinces, in Jo-RDC, $\mathrm{n}^{\circ}$ spécial, Kinshasa, 2008

- Loi organique $\mathrm{n}^{\circ} 08 / 016$ du 07 octobre 2008 portant organisation des entités territoriales et leurs rapports avec l'Etat et les provinces, inJo-RDC, ${ }^{\circ}$ spécial, Kinshasa, 2008

- Loi $\mathrm{n}^{\circ}$ 081-012 du 31 juillet 2008 portant principes fondamentaux relatifs à la libre administration des provinces ;

- Loi organique $\mathrm{n}^{\circ} 10 / 011$ du 18 mai 2010 portant fixation des subdivision territoriales à l'intérieur des provinces, in Jo-RDC, $\mathrm{n}^{\circ}$ spécial, Kinshasa, 2010

- Ordonnance loi $n^{\circ} 13 / 001$ du 23 février 2013 fixant la nomenclature des droits, la nomenclature impôts, taxes et redevances des provinces et des entités territoriales décentralisées ainsi que leurs modalités de répartition; in Jo-RDC, $\mathrm{n}^{\circ}$ spécial, Kinshasa, 2013.

- Circulaire $\mathrm{n}^{\circ} 003 / \mathrm{CAB} / \mathrm{MIN} / \mathrm{BUDGET} / 2007$ du 30 juillet 2007 portant instructions relatives à l'élaboration du budget de l'Etat pour l'exercice 2008 ;

- Arrête provincial $\mathrm{n}^{\circ} 01 / \mathrm{IAN} / 170 / \mathrm{CAB} / \mathrm{PO} / 2012$ portant édit budgétaire de l'exercice 2013 pour la province orientale, Kisangani, 2012

\section{REFERENCES}

1. Lubunga, M. H. (2007). l'impact de la décentralisation territoriale sur le développement en $R D C$, mémoire, UNIKIN, inédit

2. Niyibizi, M. (2014). Entraves à la mise en cuvre de la décentralisation financière en République Démocratique du Congo, mémoire de licence, UNIBU, Bunia, 2013-2014

3. Nkongolo, M. (2007). Problématique de la mise en cuvre de la décentralisation financière en République Démocratique du Congo, Maitrise en sciences économiques, UMNB.

4. Boro, E. L., \& Tumbuhan, M. H. P. (2016). Uji Ketahanan Beberapa Varietas Jagung Terhadap Serangan Sitophilus oryzae.

5. Kinyamba, S. S. (2012). Phénomène «Kuluna» ou le gangstérisme juvénile à Kinshasa|. Liber amicorum, 2012(1), 397.

6. Pinto, G. (1969). Méthodes des Sciences Sociales, Paris, Ed. Dalloz.

7. Pinto, R., \& Grawitz, M. (1971). Méthodes des sciences sociales: par Roger Pinto,... et Madeleine Grawitz,... 4e édition. Dalloz.

8. Otemikongo, M. (2006). Notes de cours de Méthode des recherche en sciences sociale, G3 SPA, Kisangani, UNIKIS.

9. Kyamusugulwa, P. M., \& Hilhorst, D. (2015). Power holders and social dynamics of participatory development and reconstruction: cases from the Democratic Republic of Congo. World Development, 70, 249-259.

10. Kyamusugulwa, P. M. (2014). Community-driven reconstruction in the Eastern Democratic Republic of Congo: capacity building, accountability, power, labour, and ownership. Wageningen University.

11. Kapya, K. (2014). Apport de la répartition des recettes à caractère national entre le pouvoir central et les provinces en République Démocratique du Congo: modalités et contraintes, Lubumbashi, 24.

12. KABAMBA, P. B. (2015). Décentralisation en République démocratique du Congo. Cellule d'Appui à la Politologie Africaine. 